\title{
EX PRAEFATIONE
}

EDITIONIS II.

Quaecumque interea scripta sunt de Horatio, ea, siquidem ad manus meas oculosque perlata sunt, legi in meumque usum convertere studui; in eaque re multum me debere profiteor Webero, Obbario*), Orellio, Duentzero, aliis. Kirchneri autem Novas Quaestiones Horatianas et Steineri Crucenacensis Commentationum Horatianarum specimen secundum quod non ita mature accepi, ut possem iis uti, magnopere doleo. Ne tamen meus liber doctissimorum virorum iudicio et copia omnino careat, unum saltem locum nunc inde expromere iuvat. Ac primum dicendum est, etiam Kirchnerum ad eos accedere criticos, qui Horatii carmina iam ante scholiastarum aetatem passim interpolata esse censuerunt; in iisque locis numerat (p.63) locos dudum a criticis damnatos: Carm. III, 4, 69-72; III, 11, 17 -20; III, 17, 2-5; IV, 4, 18-22; IV, 8, 17; Epist. I, 1, 56; et addit ad hos locos Epod. 9, 35. 36, quos versus cum Peerlkampio pluribus de rationibus Horatianos non esse putat, Sat. II, 7, 63-65, quos versus cur pro spuriis et suppositiciis habeat, suo se loco probaturum

*) Publice iudicium tulerunt de meo libro Weberus in Ephemeridibus Halensibus a. 1844. Nov. No. 285 sqq., Obbarius in Iahnii Ann. 1845. 7. XLIV. 3. p. 259 sqq., Rauchensteinius in Mageri libro, qui inscribitur Revue 1846 Ianuar. Februar.; quibus doctissimis viris et quod benevole de meo labore iudicarunt et quod non pauca indicarunt quae sequi deberem, maximas nunc ago gratias. 
pollicetur. Deinde addit: ,alios locos, certe antiquissimos, qui in libris Mstis nonnullis etiam vetustioribus leguntur, scholiastae ignorant, ut paucissimis fortasse exemplis, quae ipsi non viderunt, propagatos. In his numeramus octo versus illos decimae satirae praepositos: Lucili quam sis mendosus - ut redeam illuc, quos in Mss. haud paucis, non tamen, fateor, vetustissimis, reperimus: et locum I. Sat. 6, 126 fugio campum lusumque trigonem*)." De versu autem et difficillimo, et vexatissimo, Carm. IV, 8, 17, inter alia haec scripsit: „Sed propter impeditiorem in Hermanniana**) versuum transpositione verborum constructionem et lacunam, quae sic oritur, haud facile supplendam, convenit sub finem anni 1844 per litteras datas inter me summumque hunc criticum de faciliore hac transpositione: ,gaudes carminibus: carmina possumus $\mid$ Donare et pretium dicere muneris (sic Acr. Porph. muneri codd. nostri omnes) | Per quae spiritus et vita redit bonis | Post mortem ducibus. Non celeres fugae | Reiectaeque retrorsum Hannibalis minae, | Non incisa notis marmora publicis | Eius, qui domita nomen ab Africa cett." Sic omnia bene se habent: „carmina possumus donare, per quae spiritus et vita redit post mortem bonis ducibus i. e. per quae immortales fiunt. Sic pretium carminibus statim statuitur, et grata anaphora servatur: Non celeres fugae - non marmora

*) Hanc lectionem, olim a Cruquio e cod. Bland. 1. vulgatam, post huius interitum pro deperdita habitam, a nonnullis tamen viris doctis cupide srreptam nunc se in cod. Goth. 2, a repperisse ait Kirchnerus p. 23. Veram tamen lectionem rabiosi tempora signi tuetur etiam antiquissimus codex Barcellonensis, nuper ab Hauthalio diligenter descriptus (Ueber die älteste spanische Handschrift des Horaz und des Acron. Bonn. Habicht. 1847), et Cod. Bruxell. X. saec., quem vidit Schneidewinus, cf. Philolog. Tom. I. fasc. 1. p. 168.

**) Hermann. Epitom. doctr. metr. §. 578. ed. II. 
etc. Levius quod existit hyperbaton constructionis, sententia interposita et pretium dicere muneris, facile defenditur similibus locis: I. Od. 1, 3-7. II. Sat. 2, 27-29. Aliam huius totius loci ex coniectura restitutionem idem vir excell. nobiscum per litteras subtilis doctrinae plenas communicavit, quam si per eum licuerit, data occasione vulgabimus." Haec ille; ego vero, si licet contra tanta nomina meam interponere sententiam, ut nullam omnino apud Horatium interpolationem praeter octo illos versus decimae satirae praepositos agnosco, sic ne huic quidem subtilissimae transpositioni faveo cum iisque potius facio, qui, si quid non bene scriptum apud Horatium reperitur, culpam non grammaticis, non scribis, non ineptis miserisque monachis iniungunt, sed aut ipsi poetae - quem interdum etiam dormitasse cur non credamus? - aut nostrae parum rectae intellegentiae vel diverso de talibus rebus iudicio. Idem iudicare etiam Steinerum video p. 5 sq. 11. 12 sq.; quanquam quae idem vir doctissimus de explicando illo carmine magis artificiose quam veri similiter statuit, meam quidem approbationem non habent. Sic enim scripsit p. 9: „sententiarum nexus ostendit, verbis illis celeres fugae etc. non fugam ipsam sed fugam marmore expressam declarari. Itaque illa aetate Scipionis maioris monumentum Romae fuisse statuo, in quo cives et ipsum spectabant ducem egregium marmore effictum (v. 14) et res illius praeclaras sive marmore sive aereis columnis (cf. Lamprid. Alex. Sever. c. 28) repraesentatas: celeres Poenorum ex Italia fugas Hannibalisque nequiquam minitabundi atque Scipionis ultoris in Africam traiectionem (v. 16); incendia castrorum Hasdrubalis et Syphacis (Liv. 30, 5. 6. Flor. 2, 6), incendia navium Karthaginiensium (Liv.40, 43), Scipionem domita apud 
Zamam Africa in Italiam redeuntem. - Tali vero honore quis dignior Scipione? quem Ennius sic affatur: "Quantam statuam faciet populus Romanus, quantam columnam, quae res tuas gestas loquatur?" Horatii igitur aequales qui illa castrorum naviumque incendia marmore expressa vidissent, profecto cum hos versus legebant non poterant, ut nonnulli interpretes nostri, de incensa a Scipione minore Karthagine cogitare." Quae interpretatio, artificiose ut dixi excogitata, ideo non poterit probari, quod nec statuae nec columnarum in ipso carmine ulla reperitur, ne minima quidem significatio: immo totus locus de marmoribus et titulis primo ita est generalis, ut de omnibus magnis viris non possit non intellegi, deinde suo more poeta ad generalem sententiam inlustrandam sensim ad exemplum delabitur; nec, puto, ipsi Romani statuam, quae Romae fuit*), hoc carmine descriptam et comparatam repperissent. Itaque ad eiusmodi fictionem non prius licebit confugere, quam quae in eo carmine insunt difficultates non poterunt superari ratione alia ac magis probabili.

Legi Meinekianae, qua carmina omnia, et quae monostropha et quae distropha ante habebantur, in strophas quaternorum versuum dividere iubemur, ne nunc quidem obsecutus sum. De qua lege quid sentirem, in Horatianorum particula altera, a. 1845 con-

*) Vide Liv. XXXVIII, 56: „alii Romse, alii Literni et mortuum et sepultum. Utrobique monumenta ostenduntur et statuse: nam et Literni monumentum monumentoque statua superimposita fuit, quam tempestate deiectam nuper vidimus ipsi; et Romae extra portam Capenam in Scipionum monumento tres statuae sunt, quarum duae Publii et Lucii Scipionum dicuntur esse, tertia poetae Quinti Ennii." Ceterum de hoc carmine disputarunt etiam Gerberus in scriptione scholiastica, Sondershusae 1842. Lachmannus in Schneidewini philologo, Tom. I. fasc. 1. p. 164 sqq., et contra hunc acutissime $\boldsymbol{F}$. Ritterus, Bonnensis, ibid. fasc. 3. p. 581 sqq. 
scripta, p. 15 professus sum. Nunc video, ne Steinerum quidem ei legi favere; nec aliter Gerberus 1.l. p. 10 sq. Kirchneri alia ratio est; is enim, cum antea, uti fatetur p. 64, hac potissimum dubitatione deterreretur, quaenam interna huius odarum in carmina tetrastropha divisionis quasi lex esse posset, cum externa certe norma non firmiter constituta appareret, iam causam sibi ac legem repperisse visus est. Statuit enim, Horatium omnia sua carmina lyrica eo consilio composuisse eamque formam metricam iis induisse, ut ad lyram, si non omnia cantarentur, at certe cantari possent. In qua re vir doctissimus mirifice deceptus est. Nam, ut paucis exemplis defungar, carmina quae $x \alpha \tau \dot{\alpha}$

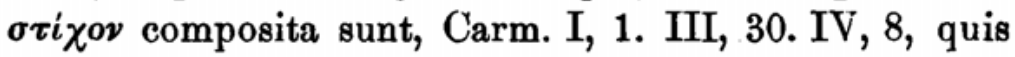
credat eo consilio ab Horatio composita esse, ut ad lyram possent cantari? Nam posse ea cantari quis negabit, quoniam omnia, etiam quae sano sensu plane carent, cantari possunt? Sed admodum mira ea esset cantatio, si severo quis vultu istius modi carmina decantare vel ita recitare conaretur, ut lyra iis praeluderet et inter singulas strophas fidium cantus interponeret. Agedum, periculum fac et sume carmen trigesimum tertii libri, quod intactum reliquerunt et Hermannus et Kirchnerus; ibi tu interpone inter singulas strophas quas volunt, i. e. post v. 4. 8.12, fidium cantus et videbis, quam violenter sententias disieceris quantamque provocaveris discrepantiam. De argumentis et rebus, quae musicae arti quam maxime possunt adversantur, nihil amplius dicam. Deinde quod alterum a Kirchnero additur argumentum, de innumeris locis, in quibus Horatius verbis utitur de lyra et cantu sumptis, ut Carm. IV, 9, 4. IV, 15, 2. I, 6, 19. I, 32, 1 sq. cet., ne hoc quidem quicquam valet. Partim enim posita sunt ea vocabula in carminibus, quorum argu- 
menta ita sunt comparata, ut et possint cantari et fortasse etiam cantarentur, partim autem nihil significant aliud nisi carmina facere, carmina componere. Hae enim dictiones exortae sunt ex antiqua poeticae artis origine, nec tamen qui poetae iis utebantur, simul indicare voluerunt, ea ipsa carmina ad fidium cantus esse accommodanda. Quae cum ita sint, ne ea quidem placet ratio, qua Kirchnerus carmen III, 12 tetrastrophon fecit,

Miserarum est neque amori

Dare ludum neque dulci

Mala vino lavere aut exanimari metuentes

Patruae verbera linguae.

Nec me paenitet solitam huius carminis in strophas ternorum versuum dividendi rationem, multo suaviorem et magis concinnam, servasse. Ceterum non possum facere, quin uno verbo dicam quam gratum mihi fuerit vidisse, etiam a Kirchnero p. 43 scholiastam jllum, quem putabant, Aemilium prorsus fictum ac nullum esse probari. Idem iam ante sex annos evincere studui in Horatianorum partic. I. Aquisgrani conscripta p. 8; idem nuper ostendit Hauthalius, vir doctissimus, in Museo Rhenano a. 1846. p. $516 \mathrm{sqq}$; hic quidem nova ratione; Kirchnerus in id ipsum incidit, quod ego iam tum proposui. Sed haec hactenus.

Embricae a. d. V. Kal. Nov. MDCCCXLVII. 


\section{EX PRAEFATIONE}

EDITIONIS III.

In emendandam novam editionem Horatii mei quantam per labores officiumque licuit curam impendi; rationem naturamque libri ne nune quidem mutavi. Nam quanquam non defuerunt qui consulenti mihi persuadere vellent, ut commentario argumentisque singulorum carminum decurtatis vel mutilatis librum contraherem in angustumque deducerem, tamen metui, si illorum vocibus auscultarem, ne minuerem simul vel demerem potius si quid esset vel probabile vel peculiare. Scio quidem, inter magistros scholarum publicarum esse aliquos, qui editiones commentariis instructas a suis lectionibus prohibeant nudaque poetae verba discipulorum manibus tradant. Quorum studium voluntatemque tantum abest ut reprehendere audeam, ut magnopere laudem, siquidem in ipsis lectionibus id possint assequi, ut viva voce doctrinaeque cura et diligentia ea omnia, quae ad veram carminum intellegentiam necessaria sunt, adulescentulorum animis ipsi praebeant memoriaeque infigant. Illud tamen metuendum est, ne ista lectio iusto saepius aut habeat aliquid vagum et incertum aut parvam tantum dulcissimi poetae partem comprehendat. Iis autem, qui commentarios ob eam causam prohibent, ne adulescentium celeritate acieque oculorum et calliditate ingeniorum inter docendum et explorandum quasi quaedam insidiae sibi struantur, optime olim Frid. Iacobsius, nuper Kruege- 
rus in praefat. edit. sermonum p. IV occurrit. Praterea cum ne summa quidem assiduitas vel magistrorum vel discipulorum eo possit pervenire, ut omnia legantur quae lectu dignissima sint, non potest fieri quin privatis studiis bona pars poematum relinquatur; quae si vult intellegi, adiumenta requirit non parca aut debilia, sed ad superandas difficultates et parata semper et firma. Deinde si licet sperare, fore ut iuventus tantopere blanditiis poetae capiatur, ut etiam relictis ludis et virili aetate ad eius lectionem sua sponte ac libenter recurrat, ubertas argumentorum adnotationumque satis habere videtur non excusationis sed necessitatis.

Editiones commentationesque, quae interim prodierunt quaeque ad me pervenerunt, legi ex iisque quaecumque meis meliora videbantur in meum usum converti. Fructus autem quem inde cepi, minor fuit quam exspectaveram*).

Codicum scripturas scholiastasque denuo diligenter consului et examinavi; quo factum est, ut antiquissimorum librorum Mss., maxime Blandiniorum Cruquii, auctoritate lectiones in prima et altera editione receptas nunc cogerer mutare. Quod quam saepe necessarium visum sit ut intellegatur, locos ipsos nunc addam: Carm. I, 7, 17. I, 8, 2. I, 15, 22. I, 19, 2. I, 24, 13 **). I, 25, 2. III, 4, 31. III, 15, 16. III, 20, 8. IV, 4, 73. C. S. 68. 71. 72. Sat. I, 3, 57. I, 4, 87. I, 6, 31. I, 7, 50. II, 1, 31. II, 2, 48. II, 2, 56. II, 2, 95. II, 3, 96. II, 3, 129. II,

*) Iuvat commemorare etiam editionem, quae hoc titulo ornata prodiit: „Horatii carmina quae exstant omnia. Iuxta editiones Doering., Orellii et Lipsianam accurate recensita. Oxonii: I. Abrams. Londini: William Allan. 1849." Quae cum nescio qualem textum praeberet, Horatii vitam et indicem metrorum ex mea editione clam sibi surripuit.

**) De hoc loco cf. Quaestiones meas Horatianas (Bonnae. Habicht 1841.) p. 48 sqq. 
3, 170. II, 3, 212. II, 3, 276. II, 4, 47. II, 6, 4. II, 6, 44. II, 6, 48. II, 8, 52. II, 8, 53. Epist. I, 1, 72. I, 2, 38. I, 3, 30. I, 13, 2. I, 15, 10. I, 16, 22. I, 18, 111. I, 19, 22. 23. II, 1, 69. II, 1, 85 (A. P. 161). II, 1, 167. II, 1, 186. II, 2, 16. II, 2, 158.

Regimontii Borussorum a.d. XIII. Kal.Mai. MDCCCLIV.

\section{PRAEFATIO \\ EDITIONIS IV.}

Quam abhinc annos prope sex aequitati doctorum hominum commendavi editionem tertiam, eius exempla cum essent divendita, institit redemptor egregius, ut quartum emitteretur libellus. Quod ut honeste fieret, denuo consului varietatem optimorum codicum, praecipue Blandiniorum Cruquii, antiquorum Bentleii et Orellii. Inde autem quem fructum ceperim ad restituenda verba poetae, intelleget qui hos locos cum superioribus editionibus comparaverit: Carm. I, 8, 2. 6 . 7. I, 15, 9. 24. I, 16, 8. III, 1, 43. III, 17, 13. III, 24, 60. III, 25, 9. IV, 1, 22. IV, 4, 31. IV, 6, 21. Epod. 11, 2. Sat. I, 5, 67. I, 7, 15. I, 10, 27. 31. 86. II, 2, 118. II, 3, 43. 259. Epist. I, 2, 32. I, 6, 57. I, 10, 24. I, 11, 16. I, 18, 16. 46. II, 1, 114 . 260. II, 2, 3. 93. 128. A. P. 46. 65. 92. 139. 153. Aliorum editiones, inter quas Meinekii alteram, Kirchneri, Doederlinii, Teuffelii, Ritteri honoris causa nomino, et commentationes de singulis vel carminibus 
vel locis scriptas, quascumque inspicere licuit, diligenter contuli; quo saepius factum est, ut in explicando poeta meliora doctus sententiam mutarem. Ceterum commentarii naturam, usu comprobatam, intactam reliqui, nisi quod criticam adnotationem ad tutandas lectiones passim auxi; tabulam chronologicam Kolsteri*) admonitionem secutus locupletavi; indicibus et nominum et rerum haud pauca addidi, quae olim praetermittenda viderentur aut recens essent recepta. Praeterea haud indignum, spero, ornamentum accedet tabula villae Horatianae, quam filius tertius, qui nunc ipsum Horatii carmina lectitare incohavit, me duce quam potuit accuratissime delineavit, Sosius honestissimus lapidi incidendam curavit. In qua re magnopere me adiuvit libellus gallice scriptus hoc titulo: Etude biographique sur Horace par A Noël des Vergers (Paris, Didot 1855); cui quae additae sunt tabulae, altera inscripta Villa d'Horace et ses environs, altera Plan topographique de la vallée de la Digentia et de la villa d'Horace, dressé par Mr. Pietro Rosa, architecte, eas quidem immutatas recipere non licuit nec si licuisset voluissem; vetuit enim tota tabularum ratio neque clara satis aut perspicua neque adulescentibus admodum habilis.

Peerlkampii in Horatii carmina gravissimi impetus id effecerunt, ut, quicumque ea sibi eripi non paterentur, quae tot saeculorum perpetua admiratio in posterum quoque videretur sacrasse, dulcissimo poetae verius intellegendo rectius interpretando acutius defendendo continuam operam navarent. Nec quicquam

*) Iahn. Annal. philol. LXXV, 7. p. 493 sqq.; praeterea publice de tertia editione iudicatum est a Tromphellero, viro doctissimo, in Mützellii mei Annal. rei scholast. 1854. Octobr. p. $776 \mathrm{sq}$. et a Meyero in libro qui inscribitur Athenée français 1854. n. 39. 
aliud efficiet Gruppii liber, qui a Peerlkampii sollertia et acumine immane quantum discrepat.

Regimontii Borussorum Idib. Sept. MDCCCLX.

\section{PRAEFATIO}

EDITIONIS V.

Ad honeste parandam quintam Horatii mei editionem meum esse vidi, et ipsius poetae versus iteratis curis subicere et adnotationes denuo diligenter examinare. Quapropter ne nunc quidem omisi, praecipuas aliorum editiones, in iisque novam Kelleri (1864) et Peerlkampii alteram (1862), accurate comparare et consulere; sed bis tantum coactus sum receptam ante lectionem mutare, reliqua recte scripsisse mihi visus sum. Etenim Carm. I, 23, 5 nunc cum Meinekio et Kellero codicum auctoritati Gogavini, Mureti et Bentleii coniecturam vepris ad ventum praetuli, et Carm. III, 3, 12 bibet scripsi, quam scripturam iam Quaest. Horat. part. I. II. (Bonnae 1841) p. 21 commendaram, sed recipere inter poetae verba non eram ausus. In adnotationibus non pauca sunt, quae corrigenda esse intellexi; qui comparaverint, ipsi videbunt quae sint vel limata vel resecta vel addita. Nec raro interductum mutavi, veluti Sat. I, 1, 95. 105. 3, 65. 4, 63. 5, 15 sqq. 7, 72. II, 6, 34. Epist. I, 6, 29. 16, 59. 17, 32 alias. Dè ordine, quo carmina Horatii per libros disposita sunt, novam nec fortasse inertem adnotationem p. $17 \mathrm{sq}$. ad- 
didi; conspectus etiam metrorum quaedam additamenta accepit.

Vratislaviae Idib. Mai. MDCCCLXVII.

\section{PRAEFATI 0}

EDITIONIS VI.

Quam explicandis Horatii operibus per septem lustra continuam navavi operam et industriam, eam ne nunc quidem, cum divendita superiorum editionum satis magna copia sextum liber esset emittendus, Venusino poetae detraxi. Luciani Muelleri, Lehrsii, Kelleri Holderique editiones comparavi, aliorum vel libros vel commentationes diligenter legi, et quicquid vel in poetae ipsius versibus vel in meis adnotationibus minus bene scriptum esset, id accurate emendare studui. Inde factum est, ut lectiones septies mutare cogerer: Carm. III, 14, 11. Sat. I, 6, 75. I, 7, 34. II, 3, 183. 216. Epist. I, 6, 35. II, 1, 198; cur ita videretur, in ipsis locis ostendi. Denique hoc quoque profitendum est, nunc me orthographiam latinam ad eam rationem, quae a Brambachio probata est, accommodasse; in qua re illud me maxime movit, quod rectores scholarum, qui ex tota hac Silesiarum provincia ante annum et dimidium Suidnitiam convenerant, prope omnes mecum consentiebant, curandum esse, ut in scribendis latinis verbis non minus quam in patriis tandem ea, qua nunc luxuriamur, licentia depelleretur; nec melius id aut rectius fieri posse, 
quam si Brambachium ducem sequentes eam amplecteremur normam, quam Quintilianus eiusque aequales constituisse viderentur.

Vratislaviae Idib. Februar. MDCCCLXXV.

\section{PRAEFATIO}

\section{EDITIONIS VII.}

De sexta Horatii mei editione multo et citius et benevolentius publice iudicatum est (cf. Mewes in Zeitschr. f. Gymnasialw. 1877. Octob. Fritsch in Bursian Jahresbericht 1875. fasc. 3 p. 184 sqq. $E$. W. in Philolog. Anzeiger 1875. p. 221. Künstler in Zeitschr. f. Philologie XVIII. fasc. 5. Hirschfelder in Zeitschr. f. Gymnasialw. XXIX. 10. p. 601 sq.), quam licuit sperare; quae autem a viris doctissimis notata vel desiderata sunt, iis ad emendandam septimam editionem libenter usus sum. Praecipue iis nunc satisfeci, qui conspectum metrorum ad eam normam transformandum esse censuerunt, quae hodie viris rerum metricarum peritis probata est, qua quidem in re quod optimum ducem sequi licuit, non possum satis laetari; cuius enim auctoritate et consilio melius uti potui quam Rossbachii, qui quid faciendum esset benigne et amice monstravit. Sed etiam ad constituenda verba poetae contuli quaecumque in novis vel editionibus vel commentationibus prolata et probata sunt; inter quos libros imprimis nominanda sunt Kelleri Epilegomena, qui liber, quanquam in eo etiam inveniuntur 
quae recipere vel probare non possis ab animo et iudicio impetrare, tamen optimae frugis plenissimus est atque in constituendo textu, quem vocamus, ubique debet in auxilium vocari. Deinde contuli etiam Krïgeri, Mülleri, Schützii novas editones, Fritschii satirarum uberrimum commentarium, Ecksteinii non omnibus sed nonnullis vel paucis potius destinatam, nitidissime ornatam editionem. Nec sine fructu haec librorum collatio fuit, nam saepius coactum me vidi ad mutandam lectionen, veluti Carm. I, 1, 31 inseres pro inseris; Carm. II, 12, 25 cum pro dum; III, 7, 4 fidei pro fide; III, 7, 20 monet pro movet; III, 14, 10 puellae ac Iam pro puellae haud; III, 17, 13 potes pro potis; III, 29, 6 Aefulae pro Aesulae; Epod. 11, 24 mollitie pro mollitia; Sat. I, 2, 25 Maltinus pro Malchinus; Sat. I, 4, 15 accipe iam pro accipiam; Sat. I, 5, 36 vatillum pro batillum; Sat. II, 2, 2, quae pro quam; Epist. I, 15, 6 scilicet ut ; Epist. II, 2, 80 contracta pro contacta; Epist. ad Pis. 101 adflent pro adsunt.

In orthographia, ut ante, Brambachium secutus sum; nam vestigia Kelleri aut Fritschii in mea editione adulescentium maxime usui parata deserenda erant, ne incerta et inconstans ratio legentium animos turbaret, cum unum idemque vocabulum variis locis varie scriberetur, vel necessitas cogeret in commentariis vulgarem scribendi rationem addere vel signis indicare, nimirum ut locus posset intellegi, veluti Fritschius scripsit Sat. I, 2, 89 captivǒs, Sat. II, 3, 28 novǒs; Sat. II, 3, 120 clàvēs; Sat. II, 7, 1 servǒs; Sat. II, 7, 80 conservǒs, et Keller. scripsit pisces (Sat. I, 3, 81), contra docet avis (aves) scribi debere Epileg. ad Sat. I, 8, 27, Epist. I, 18, 21; aequm Fritsch. Sat. I, 2, 34; dequoqueretur. Fritsch. Sat. II, 1, 74, Hol- 
der. Sat. II, 5, 52 ; sed cf. Keller. Epileg. ad II, 3, 42 p. 523, ad A. P. 186 p. 781. Scripsi tamen querellis Carm. II, 17, 1, a pro ah Carm. II, 17, 5.

In commentario emendando maximo usui fuerunt Reifferscheidti et Hertzii commentationes doctissimae, indicibus lectionum univers. Vratislav. praepositae; praeterea gratias agere debeo Hirschfeldero et Mewesio Beroliniensibus, qui benevole mecum communicarunt relationes de iis, quae de Horatio scripta sunt (Bursian, Jahresbericht 1879. p. 92-137. Zeitschr. f. d. Gymnasialw. VI. p. 238-340), et Car. Dziatzko, bibl. Vratisl. acad. praeposito doctissimo, qui usum mihi comparavit scriptionum, quae ad me non venerunt, ut Desjardins de itinere Brundisino Sat. I, 5, ubi vide, et Aemilii Baehrensii lectiones Horatianae Groning. 1880, quae magnam quidem copiam coniecturarum praebent, sed me minus alliciunt quoniam Horatium ipsum corrigere non arrogo, scribarum errores non semper video, ubi quid minus clarum videtur; praestat profiteri nos non intellegere quae obscura sunt, quam quae ipsi excogitavimus, sine haesitatione poetae obtrudere.

Indices in fine libri accurate perlegit et auxit Aug. Otto, phil. Dr. semin. reg. paedag. sodalis ordin., cui quod molestum laborem non repudiavit, gratias dico maximas. Denique hanc quoque editionem benevolentiae et aequitati doctorum hominum assidue commendo; de nonnullis locis uberius quam in commentario licuit, disserui in Annal. Berolin. (Zeitschr. f. d. Gymnasialw.) 1880. Novemb. 1881 Juni.

Scrib. Lissae prope Vratislav. a. d. VIII. Kal. Iul. MDCCCLXXXI. 
